\title{
Les membranes en crises
}

\author{
par
}

M. BUVET

Les organisateurs de cette réunion, que je tiens avant toutes choses à louer et à remercier au nom du Club Membranes d'Electricité de France, m'ont demandé d'évoquer brièvement, en fin de cette journée, les perspectives de développement des procédés à membranes en relation avec ce qu'on appelle encore aujourd'hui modestement, mais pour combien de temps encore, les Industries Agro-Alimentaires.

Je ne crois pas pouvoir mieux faire, pour situer le contexte de départ et les perspectives encore insondables de développement de ce secteur, que de reprendre l'idée qu'une émission télévisée récente, placée sous la houlette d'un acteur et chanteur célèbre, a popularisée : Vive la Crise. Ou plutôt ici : Vive les Crises ou les Membranes en Crise. A condition bien sûr de savoir se placer à la hauteur des exigences de survie que de telles crises impliquent.

Crise de l'Energie d'abord. Notre pays est pauvre, très pauvre en pétrole, et aussi en charbon. L'exploitation de son potentiel hydroélectrique, marémotricité exclue, est proche de sa saturation. Sa seule chance de survie, dès le premier choc pétrolier, et avant l'essor espéré des énergies renouvelables, a été la production à outrance d'électricité nucléaire. Mais pour affermir la mutation de sources énergétiques qu'impliquait un tel choix, il fallait l'accompagner d'un soutien à la reconversion de l'industrie, des sources thermiques d'énergie vers la source électrique. C'est la tâche qu'E.D.F. a assignée aux clubs de contact Industrie-Recherche de base, dont le Club Membranes, chargé de la promotion de l'utilisation de l'électricité en place de pétrole, dans le domaine du développement des procédés à membrane, est un exemple typique.

Crise de manque ou de reconversion d'énergie donc, mais aussi crise de surproduction agricole. Les négociations perpétuelles sur l'Europe agricole engagées à Bruxelles en sont le témoin. L'Europe, et, en Europe notre pays en tête, est en régime de pléthore de production agricole. Donc en principe de baisse des prix, sans réduction des coûts, c'est-à-dire d'instabilité prérévolutionnaire. A moins que 
les producteurs eux-mêmes, les utilisateurs actuels des productions agricoles et les titulaires actuels des marchés visés ne prennent en mains le problème et ne se mettent en quête de marchés nouveaux des productions agricoles, en quête de diversification.

L'amélioration et la diversification des produits alimentaires abordés dans cette journée en sont un premier jet évident. Mais au-delà, pourquoi pas d'autres transformations fondées sur les mêmes principes et techniques, mais à d'autres fins, des produits d'origine agricole.

Agro-Chimie fine d'abord, à finalité pharmaceutique et cosmétique par exemple, en confortant les voies déjà ouvertes vers ces marchés par les moyens issus des biotechnologies nouvelles : fermentations sur souches génétiquement modifiées, ou en réacteurs enzymatiques entre autres.

Mais aussi Agro-Chimie organique de base, plus lourde, en retour de balancier du passé. Le kilogramme de carbone issu du pétrole coûte aujourd'hui 1,5 F, demain plus encore. Et ce carbone n'est pas fonctionnalisé, il est paraffinique. Pour le fonctionnaliser, y introduire un premier point d'oxydation à partir de quoi la molécule devient plus aisément transformable, il faut dépenser autant, en chlore, électrochimique il est vrai. En regard, le coût du kilogramme de sucre, d'amidon ou cellulose se situe selon les sources et l'état du marché entre 1 et $4 \mathrm{~F}$. Ce qui place le carbone contenu, ici très, peut-être quelquefois trop, fonctionnalisé, entre 2,5 et $10 \mathrm{~F} / \mathrm{kg}$.

Ce qui signifie que dès à présent, si l'on sait mettre au point ou adapter des procédés de transformation à faible coût énergétique des poduits d'origine agricole, certains produits organiques de base ne sont économiquement plus hors de portée de la filière agricole.

Là est l'avenir, énorme, des industries de transformation issues de l'agriculture, dont les industries agro-alimentaires restent aujourd'hui le principal souvenir d'une époque, temporairement révolue par l'abondance pétrolière, mais qui doit revenir. Que nous devons faire revenir sous peine de disparition définitive de notre pays, pourtant bien placé à cet égard, du concert des nations industrialisées.

En avons-nous les moyens ? Certes personne ne niera ici que nous n'ayons au moins la capacité de les mettre en œuvre, s'il n'y manque l'espoir. Faut-il l'avoir? Y a-t-il une issue, des solutions aux multiples problèmes que pose une telle reconversion?

J'ai regretté, tout à l'heure, en réfléchissant aux arguments pour convaincre qu'il aurait été bon de produire ici, de n'avoir pas à ma disposition une série de photos prises au zoom :

- d'un athlète complet ou d'une nymphe éblouissante dans l'état de nature, pour susciter votre convoitise d'abord, au goût de chacune ou de chacun, en preuve évidemment de superbe réussite technologique des choix effectués par l'évolution, 
- d'un de leurs organes ensuite au gré de vos fantasmes préférés,

- et puis au delà, de tissus,

- et surtout de ces merveilleuses membranes, en assises pluriou monocellulaires, ou en couches bimoléculaires grâce auxquelles ces merveilleux objets fonctionnent si longtemps et si bien.

Là se trouvent, en effet, les perspectives du possible dans le développement des procédés à membranes de compositions et structures variées à l'infini pour la transformation des produits d'origine agricole. Et bien plus vastes encore si l'on imagine combien de membranes pourront être préparées en usant des ressources de la chimie de synthèse en milieu non naturel, auquel le vivant n'a pas accès.

Et cela métabolise, fabrique ses vecteurs d'énergie, ses matériaux de structure, se guérit ou se répare plus souvent seul qu'aidé, et vieillit moins vite que ne se corrodent aujourd'hui nos outils très modestement comparables.

C'est à la mesure de cet objectif, que doit se situer, dès aujourd'hui, l'ambition de ceux que nous devons à l'initiative de la S.F.F., de l'I.N.R.A., de l'E.N.S.C.R. et de l'I.U.T. de St-Nazaire, de voir réunis ici. C'est-à-dire de nous tous ou de nos successeurs ou remplaçants, si nous ne savons hisser notre volonté et poursuivre nos efforts à la hauteur de cette ambition. 